\title{
Irregular oscillatory patterns in the early-time region of coherent phonon generation in silicon
}

\author{
Yohei Watanabe, ${ }^{1}$ Ken-ichi Hino, ${ }^{2,3,{ }^{*}}$ Muneaki Hase, ${ }^{4}$ and Nobuya Maeshima ${ }^{3,2}$ \\ ${ }^{1}$ Doctoral Program in Materials Science, Graduate School of Pure and Applied Sciences, University of Tsukuba, \\ Tsukuba, Ibaraki 305-8573, Japan \\ ${ }^{2}$ Division of Materials Science, Faculty of Pure and Applied Sciences, University of Tsukuba, Tsukuba 305-8573, Japan \\ ${ }^{3}$ Center for Computational Sciences, University of Tsukuba, Tsukuba 305-8577, Japan \\ ${ }^{4}$ Division of Applied Physics, Faculty of Pure and Applied Sciences, University of Tsukuba, Tsukuba 305-8573, Japan
}

(Received 21 May 2017; published 15 September 2017)

\begin{abstract}
Coherent phonon (CP) generation in an undoped Si crystal is theoretically investigated to shed light on unexplored quantum-mechanical effects in the early-time region immediately after the irradiation of ultrashort laser pulses. We examine time signals attributed to an induced charge density of an ionic core, placing the focus on the effects of the Rabi frequency $\Omega_{0 c v}$ on the signals; this frequency corresponds to the peak electric-field of the pulse. It is found that at specific $\Omega_{0 c v}$ 's, where the energy of plasmon caused by photoexcited carriers coincides with the longitudinal-optical phonon energy, the energetically resonant interaction between these two modes leads to striking anticrossings, revealing irregular oscillations with anomalously enhanced amplitudes in the observed time signals. Also, the oscillatory pattern is subject to the Rabi flopping of the excited carrier density that is controlled by $\Omega_{0 c v}$. These findings show that the early-time region is enriched with quantum-mechanical effects inherent in the $\mathrm{CP}$ generation, though experimental signals are more or less masked by the so-called coherent artifact due to nonlinear optical effects.
\end{abstract}

DOI: 10.1103/PhysRevB.96.125204

\section{INTRODUCTION}

Coherent phonon (CP) generation is one of the representative ultrafast phenomena induced by an ultrashort pulse laser, and a great number of studies on this have been reported in diverse fields of physics and chemistry [1]. In condensedmatter systems, CPs are observed in various bulk materials and heterostructures such as semiconductors, semimetals, superconductors, and so on [2-9]. The exploration of the $\mathrm{CP}$ generation mechanism has been targeted for these experimental studies, in which observed signals are examined based on the classical model following a damped forced harmonic oscillation. In particular, an initial phase built in the oscillator with an asymptotic sinusoidal form in time is considered as a key quantity for the generation mechanism. Most of theoretical studies are devoted to analyzing this phase in either a phenomenological or a semiclassical manner [10,11].

However, such an approach has difficulty revealing quantum-mechanical effects inherent in the $\mathrm{CP}$ generation. These effects are dominant in the initial stage of $\mathrm{CP}$ dynamics in which a great number of photoexcited carriers still stay in nonequilibrium states before being relaxed; hereafter, this stage is termed as the early-time region (ETR). In fact, intricate signals observed in the ETR have been interpreted as coherent artifacts (CAs) due to nonlinear optical interference, making it difficult to extract information on the intrinsic dynamics of investigated materials $[12,13]$. It is just the transient Fano resonance that has been studied as a quantum-mechanical effect inherent in the CP generation; the detail of it has been brought to light recently $[8,14,15]$.

The aim of this paper is to explore quantum-mechanical effects still hidden in the ETR of the CP generation in undoped $\mathrm{Si}$ based on the polaronic-quasiparticle (PQ) model developed

\footnotetext{
*hino@ims.tsukuba.ac.jp
}

by the authors [15]. In this model, a PQ operator-defined as a combination of a set of pairs of electron operators and a longitudinal optical (LO) phonon operator-is introduced to describe such a transient system in which the high-density carriers generated by an intense ultrashort-pulse laser in a flash lead to a strong interaction with an LO phonon [16]; the Fano resonance mentioned above was first figured out by this model [15]. The hot carriers thus generated fulfill a vital role here, as seen below. In particular, a collective excitation mode (plasmon) resulting from the carriers is focused, and its effects on the amplitude and initial phase of the CP signal are evaluated.

The remainder of this paper is organized as follows. Section II describes the theoretical framework, Sec. III presents the results and discussion, and Sec. IV presents the conclusion. Atomic units are used throughout unless otherwise stated.

\section{THEORY}

The total Hamiltonian of this system, represented by $\hat{H}(t)$, consists of an electron Hamiltonian $\hat{H}_{e}$, an electron-laser interaction $\hat{H}^{\prime}(t)$ at time $t$, an LO phonon Hamiltonian $\hat{H}_{p}$, and a deformation-potential interaction between the electron and the LO phonon $\hat{H}_{e-p}$. That is,

$$
\hat{H}=\hat{H}_{e}+\hat{H}^{\prime}(t)+\hat{H}_{p}+\hat{H}_{e-p},
$$

where

$$
\begin{gathered}
\hat{H}_{e}=\sum_{b \boldsymbol{k}} \varepsilon_{b \boldsymbol{k}} a_{b \boldsymbol{k}}^{\dagger} a_{b \boldsymbol{k}}+\frac{1}{2} \sum_{\boldsymbol{q} \neq 0} V_{\boldsymbol{q}}^{(C)} \\
\times \sum_{b b^{\prime} \boldsymbol{k} \boldsymbol{k}^{\prime}} a_{b \boldsymbol{k}+q}^{\dagger} a_{b^{\prime} \boldsymbol{k}^{\prime}-q}^{\dagger} a_{b^{\prime} \boldsymbol{k}^{\prime}} a_{b \boldsymbol{k}}, \\
\hat{H}^{\prime}(t)=-\sum_{\boldsymbol{k}}\left[\Omega_{c v}(t) a_{c \boldsymbol{k}}^{\dagger} a_{v \boldsymbol{k}}+\Omega_{v c}(t) a_{v \boldsymbol{k}}^{\dagger} a_{c \boldsymbol{k}}\right], \\
\hat{H}_{p}=\sum_{\boldsymbol{q}} \omega_{\boldsymbol{q}} c_{\boldsymbol{q}}^{\dagger} c_{\boldsymbol{q}},
\end{gathered}
$$


and

$$
\hat{H}_{e-p}=\sum_{b, \boldsymbol{q}, \boldsymbol{k}}\left(g_{b \boldsymbol{q}} c_{\boldsymbol{q}} a_{b \boldsymbol{k}+\boldsymbol{q}}^{\dagger} a_{b \boldsymbol{k}}+g_{b \boldsymbol{q}}^{*} c_{\boldsymbol{q}}^{\dagger} a_{b \boldsymbol{k}}^{\dagger} a_{b \boldsymbol{k}+\boldsymbol{q}}\right) .
$$

Here, $a_{b \boldsymbol{k}}^{\dagger}$ and $a_{b \boldsymbol{k}}$ are creation and annihilation operators of the electron, respectively, with its energy dispersion $\varepsilon_{b k}$ at Bloch momentum $\boldsymbol{k}$ in band $b ; \varepsilon_{b \boldsymbol{k}}$ is evaluated here based on the effective-mass approximation with parabolic dispersion in the vicinity of $\Gamma$ points of the conduction band, labeled as $b=c$, and the heavy-hole valence band, labeled as $b=v$. $V_{\boldsymbol{q}}^{(C)}$ is a Coulomb potential with momentum $\boldsymbol{q} \cdot c_{\boldsymbol{q}}^{\dagger}$ and $c_{\boldsymbol{q}}$ are creation and annihilation operators of the LO phonon with its energy dispersion $\omega_{\boldsymbol{q}}$ at Bloch momentum $\boldsymbol{q}$. The externallaser electric field is given by $F(t)=f(t) \cos \omega_{0} t$, where $\omega_{0}$ represents the center frequency and $f(t)$ is a Gaussian-shaped pulse-envelope function with a peak amplitude $F_{0} \equiv f(0)$ and a temporal width $\tau_{L}$ - the full width at half maximumsatisfying $\tau_{L} \ll T_{q} \equiv 2 \pi / \omega_{q} . \Omega_{0 c v}$ is the Rabi frequency given by the product of $F_{0}$ and an electronic dipole moment $d_{c v}$ between $\Gamma$ points of $c$ and $v$ bands, namely,

$$
\Omega_{0 c v}=d_{c v} F_{0} .
$$

Further, $\Omega_{c v}(t)$ is defined as $\Omega_{c v}(t)=d_{c v} F(t)$ and $\Omega_{v c}(t)=$ $\left[\Omega_{c v}(t)\right]^{*} . g_{b q}$ is a coupling constant of the $b$-band electron ( $b=c$ and $v$ ) with the LO phonon.

The nonequilibrium carrier dynamics driven by $F(t)$ is governed by the time evolution of a composite operator,

$$
A_{\boldsymbol{q}}^{\dagger}\left(\boldsymbol{k} b b^{\prime}\right)=a_{b \boldsymbol{k}+q}^{\dagger} a_{b^{\prime} \boldsymbol{k}},
$$

representing a carrier density matrix for the transition from the $b^{\prime}$ band to the $b$ band with an anisotropic momentum distribution. The transferred momentum $\boldsymbol{q}$ is quite small, but finite in the CP generation, namely, $|\boldsymbol{q}| \neq 0$. In addition, the $t$ dependence of this operator and other operators to be defined below is omitted for the sake of simplicity, unless otherwise stated. The problem of concern is made tractable by applying the rotating wave approximation for the equation of motion of $A_{\boldsymbol{q}}^{\dagger}\left(\boldsymbol{k} b b^{\prime}\right)$, leading to the equation of motion of a transformed operator,

$$
\bar{A}_{\boldsymbol{q}}^{\dagger}\left(\boldsymbol{k} b b^{\prime}\right)=A_{\boldsymbol{q}}^{\dagger}\left(\boldsymbol{k} b b^{\prime}\right) e^{-i \bar{\omega}_{b b^{\prime}} t},
$$

where $\bar{\omega}_{c v}=\omega_{0}, \bar{\omega}_{v c}=-\omega_{0}$, and $\bar{\omega}_{b b}=0$. In fact, it is illustrated that when $t>\tau_{L} / 2$, this equation is dealt with based on the adiabatic approximation with respect to $t[15,17,18]$. Thus, the carriers even in nonequilibrium can be classified into two modes of collective excitation and single-particle excitation in an adiabatic sense, similarly to an equilibrium case. The former mode corresponds to a plasmon, and this is obtained by applying the bosonization procedure for an intraband contribution of $\hat{H}_{e}$; an exciton mode is neglected because of little contributions here. The resulting creation operator of plasmon, represented as $B_{q}^{\dagger}$, is described by a linear combination of the intraband density matrices, $A_{\boldsymbol{q}}^{\dagger}(\boldsymbol{k} b b)$ 's [19]. The creation operator of the single-particle excitation mode is represented by $A_{\boldsymbol{q}}^{\dagger}(\boldsymbol{k} b \bar{b})$ with $\bar{b} \neq b$, where the intraband contribution is neglected because $|\boldsymbol{q}| \approx 0$.

Here, for the sake of convenience, the following notations are introduced: $\hat{O}_{\boldsymbol{q} p h}=c_{\boldsymbol{q}}^{\dagger}, \hat{O}_{\boldsymbol{q}(\boldsymbol{k} b \bar{b})}=\bar{A}_{\boldsymbol{q}}^{\dagger}(\boldsymbol{k} b \bar{b})$, and
$\hat{O}_{q p l}=B_{q}^{\dagger}$, where the indices of $p h,(\boldsymbol{k} b \bar{b})$, and $p l$ represent phonon, single-particle excitation, and plasmon modes, respectively. Thus, the equation of motion of $\hat{O}_{q j}$ for $j=$ $\{p h,(\boldsymbol{k} b \bar{b}), \ldots, p l\}$ is given by

$$
-i \frac{d \hat{O}_{\boldsymbol{q} j}}{d t}=i \frac{d \hat{O}_{\boldsymbol{q} j}}{d t} \gamma_{\boldsymbol{q} j}+\left[\hat{H}(t), \hat{O}_{\boldsymbol{q} j}\right] \approx \sum_{j^{\prime}} \hat{O}_{\boldsymbol{q} j^{\prime}} \bar{Z}_{\boldsymbol{q} j^{\prime} j},
$$

where in the second equality of the right-hand side the equation concerned can be made linear with respect to a set of the operators $\left\{\hat{O}_{q_{j}}\right\}$ by adopting a factorization approximation to split four operator terms such as $a_{b_{2}, \boldsymbol{k}_{2}+\boldsymbol{q}_{2}}^{\dagger} a_{b_{2}^{\prime} \boldsymbol{k}_{2}} a_{b_{1} \boldsymbol{k}_{1}+\boldsymbol{q}_{1}}^{\dagger} a_{b_{1}^{\prime} \boldsymbol{k}_{1}}$ into a product of the operator $A_{\boldsymbol{q}_{2}}^{\dagger}\left(\boldsymbol{k}_{2} b_{2} b_{2}^{\prime}\right)$ and a single-particle density matrix $\left\langle a_{b_{1} k_{1}}^{\dagger} a_{b_{1}^{\prime} k_{1}}\right\rangle .\langle\hat{X}\rangle$ means an expectation value of operator $\hat{X}$ with respect to the ground state. In Eq. (9), $\bar{Z}_{\boldsymbol{q}}$ is a non-Hermitian matrix represented as

$$
\bar{Z}_{\boldsymbol{q}}=\left[\begin{array}{cccc}
\bar{\omega}_{\boldsymbol{q} p h} & 0 & 0 & M_{\boldsymbol{q} p h}^{*} \\
0 & \bar{\omega}_{\boldsymbol{q}(\boldsymbol{k} b \bar{b})} & 0 & M_{\boldsymbol{q}(\boldsymbol{k} b \bar{b})}^{\prime} \\
0 & 0 & \ddots & \vdots \\
M_{\boldsymbol{q} p h} & M_{\boldsymbol{q}(\boldsymbol{k} b \bar{b})} & \cdots & \bar{\omega}_{\boldsymbol{q} p l}
\end{array}\right],
$$

with $\bar{\omega}_{\boldsymbol{q} j}=\omega_{\boldsymbol{q} j}+i \gamma_{\boldsymbol{q} j}$, where $\omega_{\boldsymbol{q} p h} \equiv \omega_{\boldsymbol{q}}, \omega_{\boldsymbol{q} p l}$ represents a plasma frequency, and

$$
\omega_{\boldsymbol{q}(\boldsymbol{k} b \bar{b})} \equiv \varepsilon_{b \boldsymbol{k}+\boldsymbol{q}}^{(r)}-\varepsilon_{\bar{b} \boldsymbol{k}}^{(r)}-\bar{\omega}_{b \bar{b}} .
$$

Here, renormalized $b$-band electron energy is given by

$$
\varepsilon_{b \boldsymbol{k}}^{(r)}=\varepsilon_{b \boldsymbol{k}}-\sum_{\boldsymbol{q}} V_{\boldsymbol{q}}^{(C)} \bar{\rho}_{b b \boldsymbol{k}+\boldsymbol{q}},
$$

and $\bar{\rho}_{b b^{\prime} \boldsymbol{k}}=e^{-i \bar{\omega}_{b b^{\prime}} t}\left\langle a_{b \boldsymbol{k}}^{\dagger} a_{b \boldsymbol{k}}\right\rangle$ is a single-particle density matrix under the rotating wave approximation. $\gamma_{\boldsymbol{q} j}$ represents a damping constant of the $j$ th mode. $M_{q p h}$ represents an effective coupling between the LO phonon and plasmon modes, which is mostly determined by $g_{b \boldsymbol{q}}, V_{\boldsymbol{q}}^{(C)}$, and $\bar{\rho}_{b b \boldsymbol{k}}$. On the other hand, $M_{\boldsymbol{q}(\boldsymbol{k} b \bar{b})}$ and $M_{\boldsymbol{q}(\boldsymbol{k} b \bar{b})}^{\prime}$ represent effective couplings between the single-particle excitation and plasmon modes, which arise from an equal-time commutator $\left[\hat{O}_{q(k b \bar{b})}, \hat{O}_{q p l}\right]$, where $M_{\boldsymbol{q}(\boldsymbol{k} b \bar{b})}^{*} \neq M_{\boldsymbol{q}(\boldsymbol{k} b \bar{b})}^{\prime}$; for the detailed expressions of these effective couplings, consult Ref. [17].

Now, the PQ operator is defined as a linear combination of all of the $\hat{O}_{q j}$ 's as

$$
P_{q j}^{\dagger}=\sum_{j^{\prime}} \hat{O}_{q j^{\prime}} V_{q j^{\prime} j}^{R}
$$

The introduction of it enables us to well describe the supposition; the electron and the LO phonon are coupled so strongly in the ETR that these are considered as a composite particle, namely, a PQ. Actually, the manifestation of the transient Fano resonance observed in lightly doped $\mathrm{Si}$ is assumed to be evidence of the birth of the PQ [8], and further, the PQ model developed by the authors [15] successfully reveals the generation dynamics of this phenomenon, as mentioned in Sec. I. To be specific, the PQ is modeled according to eigenstates provided by $\bar{Z}_{\boldsymbol{q}}$, the left and right eigenvalue problems of which are given by $V_{\boldsymbol{q} j}^{L \dagger} \bar{Z}_{\boldsymbol{q}}=E_{\boldsymbol{q} j} V_{\boldsymbol{q} j}^{L \dagger}$ 
and $\bar{Z}_{\boldsymbol{q}} V_{\boldsymbol{q} j}^{R}=V_{\boldsymbol{q} j}^{R} E_{\boldsymbol{q} j}$, respectively, with the $j$ th adiabatic eigenvalue $E_{\boldsymbol{q} j}$ and the corresponding biorthogonal set of eigenvectors $\left\{V_{\boldsymbol{q} j}^{L}, V_{\boldsymbol{q} j}^{R}\right\}$ [20]. The time evolution of $P_{\boldsymbol{q} j}^{\dagger}$ is obtained by solving the associated Heisenberg equation, given by

$$
-i \frac{d P_{\boldsymbol{q} j}^{\dagger}(t)}{d t}=E_{\boldsymbol{q} j}(t) P_{\boldsymbol{q} j}^{\dagger}(t)-i \sum_{j^{\prime}} P_{\boldsymbol{q} j^{\prime}}^{\dagger}(t) X_{\boldsymbol{q} j^{\prime} j}(t),
$$

where a nonadiabatic coupling between the $j^{\prime}$ th and $j$ th states is represented as

$$
X_{q j^{\prime} j}(t)=\sum_{j^{\prime \prime}} V_{q j^{\prime} j^{\prime \prime}}^{L \dagger}(t) \frac{d V_{\boldsymbol{q} j^{\prime \prime} j}^{R}(t)}{d t} .
$$

In terms of the PQ operators, $c_{\boldsymbol{q}}^{\dagger}$ is represented as

$$
c_{\boldsymbol{q}}^{\dagger}=\sum_{j} P_{\boldsymbol{q} j}^{\dagger} V_{\boldsymbol{q} j, p h}^{L \dagger}
$$

due to $\sum_{j^{\prime}}, V_{\boldsymbol{q} j j^{\prime}}^{R} V_{\boldsymbol{q} j^{\prime}, p h}^{L \dagger}=\delta_{j, p h}$. Thus, the retarded phonon Green function $D_{q}^{R}\left(t, t^{\prime}\right)$ is given by $[15,21]$

$$
D_{q}^{R}\left(t, t^{\prime}\right)=\bar{D}_{q}^{R}\left(t, t^{\prime}\right)+\left[\bar{D}_{-q}^{R}\left(t, t^{\prime}\right)\right]^{*}
$$

where

$$
\begin{aligned}
\bar{D}_{\boldsymbol{q}}^{R}\left(t, t^{\prime}\right)= & -i\left\langle\left[c_{\boldsymbol{q}}(t), c_{\boldsymbol{q}}^{\dagger}\left(t^{\prime}\right)\right]\right\rangle \theta\left(t-t^{\prime}\right) \\
= & -i \sum_{j j^{\prime}} V_{\boldsymbol{q} p h, j}^{L}(t)\left\langle\left[P_{\boldsymbol{q} j}(t), P_{\boldsymbol{q} j^{\prime}}^{\dagger}\left(t^{\prime}\right)\right]\right\rangle \\
& \times V_{\boldsymbol{q} j^{\prime}, p h}^{L \dagger}\left(t^{\prime}\right) \theta\left(t-t^{\prime}\right),
\end{aligned}
$$

and $\bar{D}_{-q}^{R}\left(t, t^{\prime}\right)=\bar{D}_{q}^{R}\left(t, t^{\prime}\right)$. In practical calculations, it is assumed that the nonadiabatic coupling terms in the right-hand side of Eq. (14) are omitted because of negligibly small contributions in $t>\tau_{L} / 2$.

Following the linear response theory, $D_{q}^{R}\left(t, t^{\prime}\right)$ shows an induced charge density of ionic cores probed at time $t^{\prime}$ by a weak test potential with a $\delta$-function form $\delta\left(t^{\prime}\right)$. The induced charge density of ionic cores due to $\mathrm{CP}$ generation is given by

$$
Q_{q}(\tau) \equiv D_{q}^{R}\left(\tau+t^{\prime}, t^{\prime}\right)-D_{q}^{R(0)}\left(\tau+t^{\prime}, t^{\prime}\right)
$$

aside from an unimportant proportional constant with $\tau=t-$ $t^{\prime} \geqslant 0$. Here, the free phonon Green function $D_{q}^{R(0)}\left(\tau+t^{\prime}, t^{\prime}\right)$ without the pump field is subtracted because this contributes to the incoherent phonon generation. Thus, $Q_{q}(\tau)$ shows an oscillatory pattern of the CP precisely after the probe time $t^{\prime}$. Hereafter, the time of $t^{\prime}=0$ is exclusively concerned. To this end, $Q_{q}(\tau)$ is rewritten as

$$
Q_{q}(\tau)=A_{q}(\tau) \cos \left[\omega_{q} \tau+\Theta_{q}(\tau)\right],
$$

where $\Theta_{q}(\tau)$ and $A_{q}(\tau)$ represent a renormalized phase modulus $\pi$ and a transitory amplitude at $\tau$, respectively. In the large- $\tau$ limit, $Q_{q}(\tau)$ becomes a damped harmonics with $A_{\boldsymbol{q}}(\tau) \rightarrow A_{\boldsymbol{q}}^{0} e^{-\gamma_{q} h \tau}$ and $\Theta_{\boldsymbol{q}}(\tau) \rightarrow \theta_{\boldsymbol{q}}$, where the asymptotic amplitude $A_{\boldsymbol{q}}^{0}$ and the initial phase $\theta_{\boldsymbol{q}}$ are constants and $\gamma_{\boldsymbol{q} p h}$ arises from phonon anharmonicity.

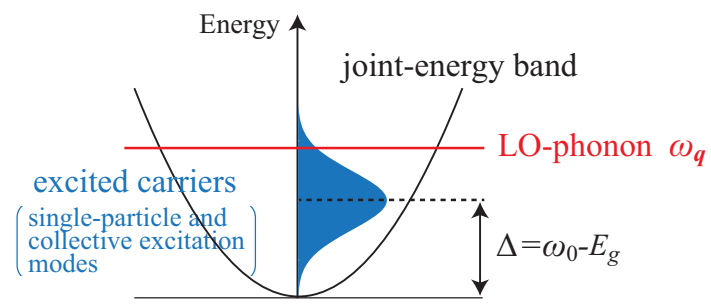

FIG. 1. The scheme of the CP generation dynamics. $\Delta=\omega_{0}-E_{g}$ represents the detuning with $E_{g}$ as the direct band gap of $\mathrm{Si}$ at the $\Gamma$ point. For more detail, consult the text.

\section{RESULTS AND DISCUSSION}

Figure 1 diagrams the scheme of the $\mathrm{CP}$ generation dynamics showing that carriers are excited by the pump pulse with detuning $\Delta$ to form the energy distribution in the joint-band energy dispersion and the energy of the LO phonon $\omega_{\boldsymbol{q}}$ is partially overlapped with it; $\Delta \equiv \omega_{0}-E_{g}$, with $E_{g}$ as the direct band gap at the $\Gamma$ point. Figures 2(a) and 2(b) show the calculated results of $\Theta_{q}(\tau)$ and $A_{q}(\tau)$,

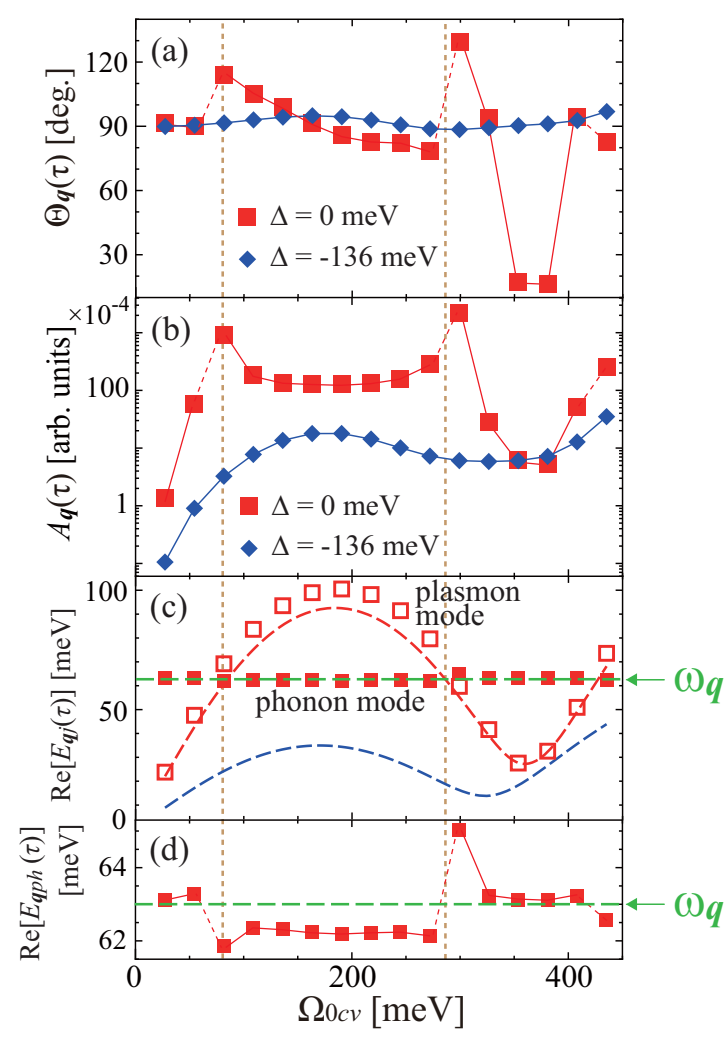

FIG. 2. (a) $\Theta_{q}(\tau)$ and (b) $A_{q}(\tau)$ as a function of $\Omega_{0 c v}$ for $\Delta=\Delta_{0}$ (red squares) and $\Delta_{-}$(blue diamonds). (c) The real parts of the adiabatic energy $E_{q j}(\tau)$ as a function of $\Omega_{0 c v}$ for $j=p h$ and $p l$; the eigenvalues mostly dominated by the phonon and plasmon modes are represented by solid and open red squares, respectively. $\omega_{q p l}(\tau)$ values for $\Delta=\Delta_{0}$ (red dashed line) and $\Delta_{-}$(blue dashed line) are also included alongside $\omega_{q}=63 \mathrm{meV}$ (green dashed line). (d) The enlarged view of panel (c) around $\operatorname{Re}\left[E_{q p h}(\tau)\right]=\omega_{q}$. In all panels, $\tau=20 \mathrm{fs}$, and the positions of $\Omega_{0 c v}^{(C 1)}$ and $\Omega_{0 c v}^{(C 2)}$ are denoted by vertical brown dotted lines. The calculated values in panels (a), (b), and (d) are connected by solid and dashed lines in order to aid the presentation. 
respectively, at $\tau=20 \mathrm{fs}$ in the ETR as a function of $\Omega_{0 c v}$. Here, material parameters given in Ref. [15] are employed, and $\tau_{L}=10 \mathrm{fs}$ corresponds to the spectral width of the laser of $370 \mathrm{meV}$. It is seen that both $\Theta_{q}(\tau)$ and $A_{q}(\tau)$ for $\Delta=\Delta_{0} \equiv 0 \mathrm{meV}$ change in an irregular manner with cusp structures at $\Omega_{0 c v}=\Omega_{0 c v}^{(C 1)} \equiv 82 \mathrm{meV}$ and $\Omega_{0 c v}^{(C 2)} \equiv 286 \mathrm{meV}$, and the envelopes of both functions change steeply around $\Omega_{0 c v}=350 \mathrm{meV}$. This contrasts with the behavior of $\Theta_{q}(\tau)$ and $A_{q}(\tau)$ for $\Delta=\Delta_{-} \equiv-136 \mathrm{meV}$, showing even more moderate alteration over $\Omega_{0 c v}$.

To deepen the understanding of this result, the real parts of adiabatic energy $E_{\boldsymbol{q} j}(\tau)$ as a function of $\Omega_{0 c v}$ are evaluated, as shown in Fig. 2(c), where solid and open red squares represent the eigenvalues mostly dominated by the phonon $(j=p h)$ and the plasmon $(j=p l)$, respectively. The plasma frequency $\omega_{q p l}(\tau)$ in proportion to the square root of the whole excited carrier density is also included for $\Delta=\Delta_{0}$ and $\Delta_{-}$in addition to $\omega_{\boldsymbol{q}}$. It is evident that $\omega_{\boldsymbol{q} p l}(\tau)$ for $\Delta=\Delta_{0}$ coincides with $\omega_{\boldsymbol{q}}$ at $\Omega_{0 c v}=\Omega_{0 c v}^{(C 1)}$ and $\Omega_{0 c v}^{(C 2)}$, leading to anticrossings between $\operatorname{Re} E_{\boldsymbol{q} p h}(\tau)$ and $\operatorname{Re} E_{\boldsymbol{q} p l}(\tau)$.

The detail of this phenomenon is shown in the enlarged figure of Fig. 2(d). The difference of $\operatorname{Re} E_{\boldsymbol{q} p h}(\tau)$ from $\omega_{\boldsymbol{q}}$ represents the self-energy resulting mostly from the interaction of the phonon with the plasmon; the contribution of the single-particle excitation mode is found negligibly small. The sharp change of the self-energy for $\Delta=\Delta_{0}$ seen at $\Omega_{0 c v}=$ $\Omega_{0 c v}^{(C 1)}$ and $\Omega_{0 c v}^{(C 2)}$ is in harmony with the manifestation of anomalous cusp structures stated above. Thus, it is concluded that the irregularity revealed here in both $\Theta_{q}(\tau)$ and $A_{q}(\tau)$ is unequivocally due to the anticrossings caused by the energetically resonant interaction of the phonon with the plasmon induced by laser pulse irradiation. According to Fig. 2(d), the plasmon-phonon interaction remains effective in the range of $\Omega_{0 c v}$ of $\left[\Omega_{0 c v}^{(C 1)}, \Omega_{0 c v}^{(C 2)}\right]$. On the other hand, there is no such irregularity for $\Delta=\Delta_{-}$because of $\omega_{q}>\omega_{q p l}(\tau)$ within the concerned range of $\Omega_{0 c v}$.

Figure 2(c) also shows that $\omega_{q p l}(\tau)$ oscillates with an approximate period of $350 \mathrm{meV}$. This is due to the interband Rabi flopping of the excited carriers that ends at $t \approx \tau_{L} / 2$, since the rough estimate of the $2 \pi$ pulse is $\Omega_{0 c v}=\Omega_{0 c v}^{(2 \pi)} \equiv$ $388 \mathrm{meV}$ besides the Coulomb correction; that of the $\pi$ pulse is $\Omega_{0 c v}^{(\pi)} \equiv \Omega_{0 c v}^{(2 \pi)} / 2$. Thus, the obvious changes of $\Theta_{q}(\tau)$ and $A_{q}(\tau)$ around $\Omega_{0 c v}^{(2 \pi)}$ for $\Delta=\Delta_{0}$ arise from the Rabi oscillation [see Figs. 2(a) and 2(b)].

Figures 3(a) and 3(b) show the calculated results of $\theta_{\boldsymbol{q}}$ and $A_{\boldsymbol{q}}^{0}$ as a function of $\Omega_{0 c v}$, respectively. The Rabioscillatory patterns still remain in both $\theta_{\boldsymbol{q}}$ and $A_{\boldsymbol{q}}^{0}$ for $\Delta=\Delta_{0}$ around $\Omega_{0 c v}=\Omega_{0 c v}^{(2 \pi)}$, while the cusp structure disappears since the plasmon-phonon interaction vanishes out of the ETR. The experimental data are also included in Fig. 3(a), showing the dependence of $\theta_{\boldsymbol{q}}$ on the pump fluence [9]. With the increase of the fluence, $\theta_{\boldsymbol{q}}$ changes from $90^{\circ}$ to the vicinity of $0^{\circ}$, which is in accordance with the calculated results for $\Delta=\Delta_{0}$.

Figures 4(a)-4(d) show the alteration of $Q_{q}(\tau)$ as a function of $\tau$ in the ETR for $\Delta=\Delta_{0}$ at the four values of $\Omega_{0 c v}$, namely, $\Omega_{0 c v}^{(C 1) \prime} \equiv 81.6 \mathrm{meV}, \Omega_{0 c v}^{(\pi) \prime} \equiv 190.4 \mathrm{meV}, \Omega_{0 c v}^{(C 2) \prime} \equiv 299.2 \mathrm{meV}$, and $\Omega_{0 c v}^{(2 \pi)^{\prime}} \equiv 353.6 \mathrm{meV}$, in the vicinity of $\Omega_{0 c v}^{(C 1)}, \Omega_{0 c v}^{(\pi)}, \Omega_{0 c v}^{(C 2)}$, and $\Omega_{0 c v}^{(2 \pi)}$, respectively [18]. Here, the number of excited

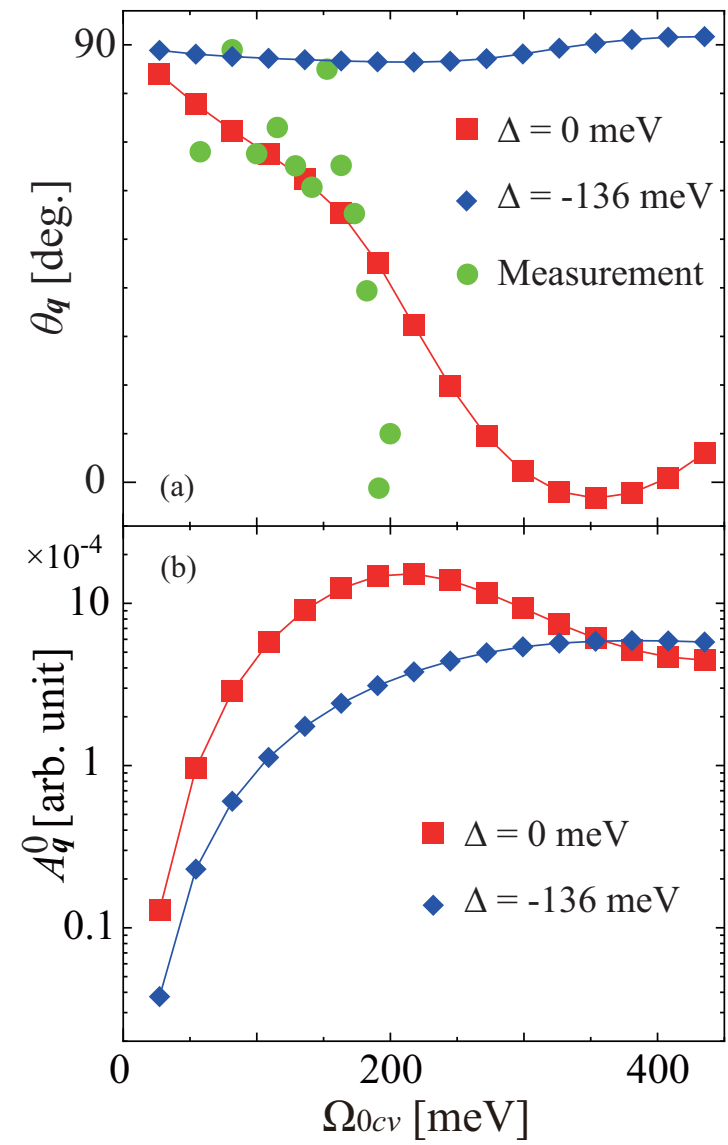

FIG. 3. (a) $\theta_{q}$ and (b) $A_{q}^{0}$ as a function of $\Omega_{0 c v}$ for $\Delta=\Delta_{0}$ (red squares) and $\Delta_{-}$(blue diamonds) along with the experimental data of $\theta_{\boldsymbol{q}}$ (green circles) [9].

carriers is mostly maximized and minimized at $\Omega_{0 c v}^{(\pi)^{\prime}}$ and $\Omega_{0 c v}^{(2 \pi)^{\prime}}$, respectively. At $\Omega_{0 c v}=\Omega_{0 c v}^{(C 1) \prime}$ and $\Omega_{0 c v}^{(C 2) \prime}$, it is seen that $Q_{q}(\tau)$ 's show marked irregularity due to the resonant interaction of the plasmon with the phonon from a simple harmonics with the period $T_{q}=66 \mathrm{fs}$. In particular, it is noted that the transitory amplitudes $A_{q}(\tau)$ at both $\Omega_{0 c v}$ 's are about ten times greater than that at $\Omega_{0 c v}=\Omega_{0 c v}^{(\pi) \prime}$, while in contrast, the asymptotic amplitudes $A_{\boldsymbol{q}}^{0}$ at the former $\Omega_{0 c v}$ 's are several times less than that at the latter one [see Fig. 3(b)]. Further, the renormalized phase $\Theta_{q}(\tau)$ shows an anomalous oscillatory pattern, especially, at $\Omega_{0 c v}=\Omega_{0 c v}^{(C 1) \prime}$, where this phase changes rapidly over $2 \pi$ around $\tau=10$ fs due presumably to the manifestation of strong anticrossing. Also, it is seen that $Q_{q}(\tau)$ is modulated by the maximized carrier inversion at $\Omega_{0 c v}=\Omega_{0 c v}^{(\pi)^{\prime}}$. Unlike it, at $\Omega_{0 c v}=\Omega_{0 c v}^{(2 \pi) \prime}$, both $\Theta_{q}(\tau)$ and $A_{q}(\tau)$ remain almost unaltered and are gradually close to the respective asymptotes; the resulting $Q_{q}(\tau)$ just shows a damped harmonic oscillation almost in the whole $\tau$ region.

In addition to the transient Fano resonance revealed in the region of $\tau \lesssim T_{q}$ [8], the two more quantummechanical effects-the plasmon-phonon resonance and the Rabi flopping-are uncovered in the ETR. As long as $\tau \geqslant \tau_{L} / 2$ [18], the induced carriers vary with respect to $\tau$ slowly enough in the ETR that the plasmon mode can 


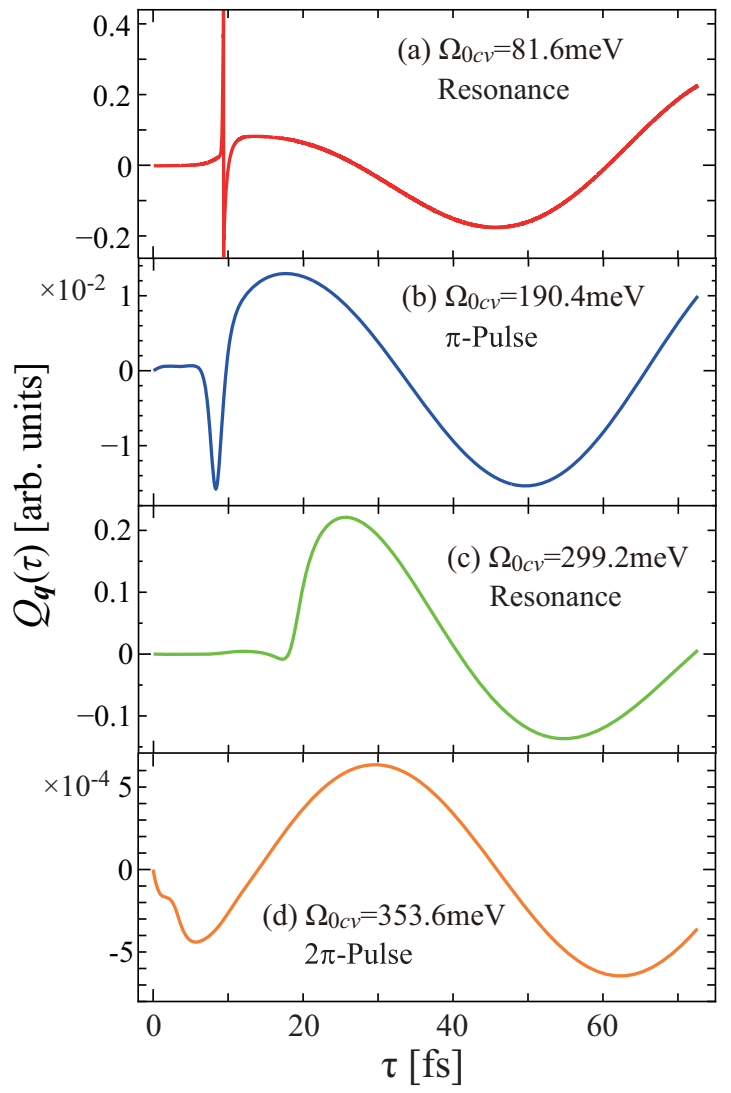

FIG. 4. $Q_{q}(\tau)$ as a function of $\tau$ in the ETR at the specific $\Omega_{0 c v}$ 's given in panels (a)-(d) with $\Delta=\Delta_{0}$.

be created instantaneously. Therefore, the resonance effect of concern differs from the delayed formation of the LOphonon-plasmon coupled modes observed out of the ETR in undoped GaAs $[2,22]$. Further, the irregular transitory signals manifested in the ETR seen in Figs. 4(a)-4(c) are distinct from CAs arising from interference during the overlap of the pump and probe pulses $[12,13]$. In particular, in the former, $Q_{q}(\tau)$ is amplified by the formation of anticrossing due to the plasmon-LO-phonon interaction, while, in the latter, the observed signal is due to the intrinsic characters of the laser cavity. As regards the Rabi flopping, the significance of it in $\mathrm{CP}$ dynamics has been overlooked thus far, though it has been pointed out just regarding CAs [23].

It is worthwhile to remark on the validity of the model adopted here for $\mathrm{CP}$ generation from the aspect of the band structure of $\mathrm{Si}$; this is subject to the diamond crystal structure. First, the electronic-energy band structure is more complicated than the simple two-band parabolic dispersion model-consisting of the $c$ band and $v$ band (heavy-hole band)-assumed in the present calculations. As regards the plasmon mode highlighted here, joint-band energy dispersions of the $c$ band with light-hole and spin-orbit split-off hole bands-referred to as $v^{\prime}$ and $v^{\prime \prime}$ bands, respectively—would also contribute somewhat to the formation of the plasmon in addition to the $v$ band. Let the joint-band energy dispersion between the $c$ band and the $b_{v}$ band be represented as

$$
\epsilon_{k}^{\left(c b_{v}\right)}+E_{g} \equiv \varepsilon_{c k}^{(r)}-\varepsilon_{b_{v} k}^{(r)},
$$

with $b_{v}=v, v^{\prime}$, and $v^{\prime \prime}$ [see Eq. (12)]. An electron state with momentum $\boldsymbol{k}$ satisfying

$$
\epsilon_{k}^{\left(c b_{v}\right)} \lesssim 1 / \tau_{L}
$$

contributes to real carrier excitation due to an opaque interband transition, where the pulse width is represented as $1 / \tau_{L}$ and $\Delta=\Delta_{0}$ is assumed for the sake of simplicity. Thus, a hole band with a greater effective mass has more contribution to the excitation, since electronic states up to larger $\boldsymbol{k}$ come into play. Actually, the $v$ band mostly governs the plasmon formation, and the $v^{\prime}$ band contributes to it to a certain extent, whereas the $v^{\prime \prime}$ band can be neglected. This is because average effective masses of $v, v^{\prime}$, and $v^{\prime \prime}$-bands are $0.523 m_{e}$, $0.154 m_{e}$, and $0.24 m_{e}$, respectively, with $m_{e}$ being the mass of the electron $[24,25]$. Further, the spin-orbit splitting of the valence bands is $440 \mathrm{meV}$ greater than $1 / \tau_{L}$ [24], while the $v$ and $v^{\prime}$ bands are degenerate at the $\Gamma_{25^{\prime}}$ point. Therefore, it is stated that the $v$-band electrons in the vicinity of this critical point are most dominant for the plasmon formation.

Second, beyond the scope of the parabolic dispersion model adopted here, CP signals arise from a lot of carrier transitions along the $\Lambda$ and $\Delta$ directions in addition to the abovementioned $\Gamma-\Gamma$ transition in the actual experiments [8]. The energy dispersions of the $c$ band along the $\Lambda$ (L valley) direction and the $\Delta$ (X valley) direction are almost parallel to the energy dispersion of the $v$ band. Thus, the joint-band energy dispersion between the $c$ band and the $v$ band is almost dispersionless, namely, independent of $\boldsymbol{k}$, and $\epsilon_{\boldsymbol{k}}^{(c v)} \approx 0$ [15]. Thus, it is stated that just the interband transition near the $\Gamma$ point produces $\boldsymbol{k}$-dispersive electron-hole continuum states, thereby resulting in the collective excitation mode of the plasmon.

Third, as regards the phonon band structure, the LO phonon mode is degenerate with the transverse-optical (TO) phonon mode at the $\Gamma_{25^{\prime}}$ point, and thus the latter mode also contributes to the $\mathrm{CP}$ signal with the same asymptotic frequency as $\omega_{q}$ for the former mode exclusively examined here. A deformationpotential interaction between the electron and the TO phonon is represented as an expression similar to Eq. (5). Unlike the LO phonon, however, the TO phonon is not coupled with the plasmon mode characteristic of a longitudinal wave and is excited just by an interaction with an intraband single-particle excitation mode of the electron [26,27]. As mentioned in Sec. II, such an intraband contribution is less dominant than the interband contribution given by $A_{q}^{\dagger}(\boldsymbol{k} b \bar{b})$ in the $\mathrm{CP}$ generation due to $|\boldsymbol{q}| \approx 0$. Thus it is stated that the anomalous CP signals observed here are governed mostly by the LO phonon. In passing, as regards the observation of TO phonons, this has been reported recently in a GaAs crystal that is coherently excited by broadband terahertz (infrared) pulses via direct coupling [28].

From the above, it is seen that the substantial result regarding the manifestation of the irregular oscillations in the observed time signals is still valid in more sophisticated models beyond the model adopted here. Thus, this result seems unlimited in actual measurements.

\section{CONCLUSIONS}

The two quantum-mechanical effects of the resonant plasmon-LO-phonon interaction and the Rabi flopping are disclosed. It is found that the underlying physics of the 
CP dynamics in the ETR is enriched by these effects. In particular, the former effect stands out, causing the striking cusp structures in $\Theta_{q}(\tau)$ and $A_{q}(\tau)$. Due to the irregular alteration of both $\Theta_{q}(\tau)$ and $A_{q}(\tau)$ with respect to $\tau, Q_{q}(\tau)$ shows the anomalous oscillatory pattern just observed in the ETR. It is expected that such effects are confirmed by experiments by minimizing the masking effect due to CAs, for instance, using orthogonal polarizations of the pumping and probing radiation. More practically, a combination of few-cycle laser pulses in the near-infrared (or visible) region with attosecond extreme ultraviolet pulses may enable us to monitor the ETR dynamics without CAs [29]. Since the Fröhlich interaction for a polar semiconductor is much greater than the deformation-potential interaction examined here, the quantum-mechanical effects described in the present study will be more enhanced in GaAs than in Si [30]. Further, these effects will be applicable for other attractive systems, such as $\mathrm{SiC}$ [31] and diamond [32]. On the other hand, the Rabi flopping is discernible in experiments by measuring the asymptotes of $\theta_{\boldsymbol{q}}$ and $A_{\boldsymbol{q}}^{0}$ as a function of $\Omega_{0 c v}$ up to more than $\Omega_{0 c v}^{(2 \pi)}$.

\section{ACKNOWLEDGMENT}

This work was supported by JSPS KAKENHI Grants No. JP23540360 and No. JP15K05121.
[1] Coherent Vibrational Dynamics, edited by S. De Silvestri, G. Cerullo, and G. Lanzani (CRC Press, New York, 2007).

[2] A. V. Kuznetsov and C. J. Stanton, in Ultrafast Phenomena in Semiconductors, edited by K. T. Tsen (Springer-Verlag, Berlin, 2001), Chap. 7.

[3] A. J. Sabbah and D. M. Riffe, Phys. Rev. B 66, 165217 (2002).

[4] S. Yoshino, G. Oohata, and K. Mizoguchi, Phys. Rev. Lett. 115, 157402 (2015).

[5] M. Hase, J. Demsar, and M. Kitajima, Phys. Rev. B 74, 212301 (2006).

[6] K. Ishioka, M. Kitajima, and O. V. Misochko, J. Appl. Phys. 103, 123505 (2008).

[7] J. J. Li, J. Chen, D. A. Reis, S. Fahy, and R. Merlin, Phys. Rev. Lett. 110, 047401 (2013).

[8] M. Hase, M. Kitajima, A. M. Constantinescu, and H. Petek, Nature (London) 426, 51 (2003).

[9] M. Hase, M. Katsuragawa, A. M. Constantinescu, and H. Petek, Nat. Photon. 6, 243 (2012).

[10] D. M. Riffe and A. J. Sabbah, Phys. Rev. B 76, 085207 (2007).

[11] Y. Shinohara, K. Yabana, Y. Kawashita, J.-I. Iwata, T. Otobe, and G. F. Bertsch, Phys. Rev. B 82, 155110 (2010).

[12] Z. Vardeny and J. Tauc, Opt. Commun. 39, 396 (1981).

[13] P. Borri, F. Romstad, W. Langbein, A. E. Kelly, J. Mork, and J. M. Hvam, Opt. Express 7, 107 (2000).

[14] D. M. Riffe, Phys. Rev. B 84, 064308 (2011).

[15] Y. Watanabe, K.-i. Hino, M. Hase, and N. Maeshima, Phys. Rev. B 95, 014301 (2017), and references therein.

[16] P. Gaal, W. Kuehn, K. Reimann, M. Woerner, T. Elsaesser, and R. Hey, Nature (London) 450, 1210 (2007).

[17] See Supplemental Material at http://link.aps.org/supplemental/ 10.1103/PhysRevB.96.125204 for the detail of the theoretical framework.

[18] Because of a steep change of the pulse envelope function $f(t)$ with respect to $t$, the adiabatic approximation supposed in Ref. [15] is not always correct in $|t| \leqslant \tau_{L} / 2$.
[19] H. Haug and S. W. Koch, Quantum Theory of the Optical and Electronic Properties of Semiconductors, 5th ed. (World Scientific, Singapore, 2009), Chaps. 1 and 12.

[20] The PQ operator thus introduced differs from that defined in Ref. [15] in that, in the latter, this operator is determined in a proper manner based on the Fano problem pertinent to the multichannel scattering problem, while in the former such a scattering problem to be solved is replaced by the more tractable eigenvalue problem just for the sake of simplicity.

[21] W. Schäfer and M. Wegener, Semiconductor Optics and Transport Phenomena (Springer-Verlag, Berlin, 2002), Chaps. 2, 10, and 11.

[22] R. Huber, F. Tauser, A. Brodschelm, M. Bichler, G. Abstreiter, and A. Leitenstorfer, Nature (London) 414, 286 (2001).

[23] M. V. Lebedev, O. V. Misochko, T. Dekorsy, and N. Georgiev, J. Exp. Theor. Phys. 100, 272 (2005).

[24] P. Y. Yu and M. Cardona, Fundamentals of Semiconductors, 4th ed. (Springer-Verlag, Berlin, 2010), Chaps. 2 and 3.

[25] C. S. Wang and B. M. Klein, Phys. Rev. B 24, 3393 (1981).

[26] K. J. Yee, K. G. Lee, E. Oh, D. S. Kim, and Y. S. Lim, Phys. Rev. Lett. 88, 105501 (2002).

[27] S. J. Sheih, K. T. Tsen, D. K. Ferry, A. Botchkarev, B. Sverdlov, A. Salvador, and H. Morkoc, Appl. Phys. Lett. 67, 1757 (1995).

[28] Z. Fu and M. Yamaguchi, Sci. Rep. 6, 38264 (2016).

[29] M. Schultze, K. Ramasesha, C. D. Pemmaraju, S. A. Sato, D. Whitmore, A. Gandman, J. S. Prell, L. J. Borja, D. Prendergast, K. Yabana, D. M. Neumark, and S. R. Leone, Science 346, 1348 (2014).

[30] N. Hayashida, Y. Watanabe, K. Hino, M. Hase, and N. Maeshima (unpublished).

[31] K. Kato, K. Oguri, A. Ishizawa, H. Nakano, and T. Sogawa, J. Appl. Phys. 111, 113520 (2012).

[32] K. Ishioka, M. Hase, K. Kitajima, and H. Petek, Appl. Phys. Lett. 89, 231916 (2006); 92, 019903 (2008). 